\title{
DESIGUALDAD SALARIAL POR GÉNERO EN EL EMPLEO DE LAS TECNOLOGÍAS DE LA INFORMACIÓN Y LA COMUNICACIÓN: EL CASO ESPAÑOL
}

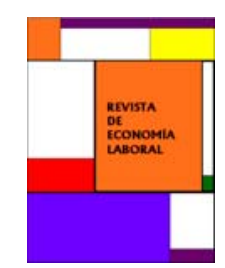

Carlos Iglesias* $\downarrow \neq$, Raquel Llorente* $\ddagger 1$, Diego Dueñas* $\ddagger$

*Instituto de Análisis Económico y Social (IAES)

$\dagger$ Universidad de Alcalá de Henares; $\ddagger$ Universidad Autónoma de Madrid

Recibido el 15 de octubre de 2009; aceptado el 10 de diciembre de 2010

\section{Resumen}

Ante la intensa difusión de las Tecnologías de la Información y la Comunicación (TIC) en España, el objetivo del artículo es determinar hasta qué punto dichas tecnologías mejoran la posición laboral femenina, reduciendo la desigualdad salarial por género. Utilizando datos de la Encuesta de Población Activa (EPA) y la Encuesta de Condiciones de Vida (ECV) se desarrollan análisis (descomposición de Oaxaca) con los que conocer cuál es el gap salarial existente por género en el empleo TIC. Los resultados obtenidos demuestran que aunque las mujeres empleadas en las ocupaciones TIC reciben salarios superiores al resto de mujeres, persisten diferencias notables con el colectivo homónimo masculino, observándose un importante componente de discriminación.

Palabras clave: TIC, género, ocupaciones laborales, salarios, desigualdad.

Clasificación JEL: J16, J31, O33.

\begin{abstract}
Given the intense diffusion of information technologies and communication (ICT) in Spain, the aim of article is to known what extent these technologies improve the position of women in labour marker, by reducing gender wage inequality. Based on data provided by the Spanish Labour Force Survey (EPA) and Living Conditions Survey (ECV), some analyses (Oaxaca decomposition) are carried out to know what the gender wage gap in ICT. The results show that while women employed in ICT occupations receive wages above the other women there are still major differences with the men in the same situation, so that an important discrimination component is observed.
\end{abstract}

Key words: ICT, gender, labour skills, Oaxaca, wages, inequality.

JEL Classification: J16, J31, O33.

1 Autora para correspondencia: raquel.llorente@uam.es. El contenido de este artículo se basa en algunos de los resultados obtenidos en el proyecto de investigación "Desajuste entre participación y posición de las mujeres en la investigación y el empleo TIC" , financiado por el Ministerio de Industria, Plan Avanza, Programa de Igualdad de Género 2007-2008, llevado a cabo por la Universitat Oberta de Catalunya, la Universidad Complutense de Madrid y el Instituto de Análisis Económico y Social (IAES) de la Universidad de Alcalá. Los autores agradecen los comentarios y sugerencias recibidas al respecto en el III Congreso Nacional sobre Mercado de Trabajo y Relaciones Laborales (Palencia) y en el 5th European Symposium on Gender and ICT (University of Bremen).

(C) Revista de Economía Laboral 


\section{Introducción}

El artículo tiene como objetivo determinar en qué medida el empleo más estrechamente relacionado con las Tecnologías de la Información y la Comunicación (TIC) es capaz de reducir en España las diferencias laborales existentes por género, verificando, más concretamente, si la discriminación salarial por género es menor o no en el empleo TIC. La difusión e importancia de las nuevas Tecnologías de la Información y la Comunicación (TIC) en el mercado laboral español resulta un hecho constatado. Durante la última fase expansiva de la economía española (1996-2007), el empleo en nuestro país se ha incrementado en 7,5 millones de personas (60 por ciento). En este contexto, una parte importante de esta creación de empleo se produjo en actividades relacionadas con el uso y producción de TIC, que experimentó un crecimiento superior a 2,9 millones de personas, hecho que resultó especialmente intenso en relación con los denominados Servicios Intensivos en Conocimiento (KIS) (Dueñas, Iglesias y Llorente, 2009). No obstante, dada la forma en que se define empleo TIC (OCDE, 2002, Eurostat, 2006), en el mismo coexisten muy diferentes tipos de trabajos en función de parámetros tales como su cualificación y productividad, por ejemplo. El debate existente entre la creación o destrucción de empleo que conlleva el desarrollo tecnológico ya ha sido superado, demostrando que los efectos directos e indirectos del cambio tecnológico digital resultan positivos y favorables en la creación de empleo, el aumento de la productividad y los salarios (Torrent-Sellens, J., 2008; OCDE, 2007) ${ }^{2}$.

Por otra parte, son bien conocidas las diferencias laborales existentes en nuestro mercado de trabajo por razones de género en contra de la población femenina, que encuentran su expresión en la totalidad de parámetros laborales: menores tasas de actividad y empleo, mayores tasas de desempleo, mayores niveles de parcialidad y temporalidad, y más frecuentes situaciones de sobrecualificación. En este sentido, las desigualdades salariales por género y su posible explicación en términos de discriminación revisten probablemente una especial relevancia.

Una idea ampliamente presente en la literatura económica y sociológica, y que vendría a relacionar los anteriores dos argumentos, es la de que las TIC ofrecen nuevas oportunidades para mejorar la posición laboral de la mujer, en la medida en que reducen el uso de cualificaciones

\footnotetext{
2 No obstante, trabajos como los de Acemoglu, D. (2002) muestran que en determinados casos como cuando el progreso tecnológico se encuentra sesgado hacia determinadas ocupaciones o cualificaciones pueden generar una pérdida de empleo en diferentes grupos poblacionales.
} 
manuales y la importancia del esfuerzo físico a favor del conocimiento, el trabajo en equipo y las habilidades de comunicación (WWW-ICT, 2004), cambiando la distribución sectorial del empleo, los requerimientos educativos de los puestos de trabajo y estimulando la demanda de cualificaciones de naturaleza no manual (Collechia and Papaconstantinou, 1996; Ducatel and Burgleman, 1999; Carnoy, 2002). De hecho, Derek H. C. Chen (2004) demuestra que la existencia o el desarrollo de un importante nivel de infraestructuras relacionadas con las TIC posibilita a nivel internacional una mayor igualdad por género tanto desde el punto de vista de la educación como del empleo.

El artículo utiliza dos fuentes de información estadística. Los datos relativos a las características del empleo femenino en el empleo TIC proceden de la Encuesta de Población Activa (EPA), mientras que para los análisis salariales se recurre a la información de la Encuesta de Condiciones de Vida (ECV) de 2008.

La ECV en una base de datos cuyo objetivo general es la producción de información sobre renta y condiciones de vida, en términos comparables para el conjunto de los países europeos. En lo que se refiere a rentas, la ECV aporta una aproximación muy rica, tanto de salarios como de fuentes de rentas no salariales, con detalle de sus distintos componentes. A nuestro juicio, la ECV (Encuesta de Condiciones de Vida) presenta algunas ventajas respecto de la Encuesta de Estructura Salarial (EES), que sería la alternativa, para los objetivos del trabajo. Por una parte, su mayor actualidad, dado que su último dato disponible corresponde a 2008, mientras que la EES más reciente es del año 2007. Por otra, aunque no se requiera dentro de este trabajo, con esta encuesta existe la posibilidad de comparación a nivel internacional. Especialmente remarcable es el hecho de que la ECV supera alguna de las carencias asociadas a la población estudiada por la EES, en la medida en que esta última fuente no incorpora sino asalariados (no considerando por tanto a los autoempleados) que trabajan en empresas de más de 10 asalariados. Tampoco considera el sector primario ni el trabajo doméstico. Además, la ECV tiene como unidad de muestreo la vivienda y los individuos que allí habitan, y no la empresa, lo que también parece más conveniente.

Para alcanzar el objetivo planteado el artículo se organizará de la siguiente manera. En primer lugar se revisa la literatura existente en relación a este tema (apartado 2). Posteriormente, en el apartado 3 se presentan una serie de datos estadísticos que muestran la posición laboral y salarial de la mujer empleada en las ocupaciones relacionadas con las TIC en España. En el apartado 4 se desarrollan una serie de modelos que permiten descomponer las diferencias salariales observadas entre hombres y mujeres en diferentes ámbitos ocupacionales, con el fin de 
validar si las mujeres empleadas en las ocupaciones TIC cuentan con un trato diferencial respecto a sus salarios. Por último, se recopilan aquellos resultados más relevantes en el apartado dedicado a conclusiones.

\section{Planteamiento general y metodológico}

Cabe afirmar que en España, el desarrollo y difusión de las TIC comenzó su despegue en el año 2000 (Lopez, S. 2005). Si bien hoy en día no alcanzamos la difusión existente en otros países europeos, el ritmo de crecimiento y asimilación en España ha sido espectacular. Desafortunadamente los efectos positivos que presentan la difusión de las TIC no se distribuyen de manera equitativa entre hombres y mujeres, apareciendo la conocida brecha digital. Las mujeres cuentan con una menor presencia dentro de las TIC (OIT, 2001) que no se termina de explicar, sobre todo teniendo en cuenta el mayor capital humano adquirido por las mujeres en los últimos años. Existe alguna evidencia, sin embargo, de que la situación laboral femenina mejora considerablemente al desarrollar actividades TIC o utilizar dicha tecnología en sus puestos de trabajo. Diaz-Santos (2008), con datos de la Encuesta de Estructura Salarial (EES) de 2002, demuestra que las diferencias salariales existentes por género se reducen considerablemente dentro de las industrias intensivas en el uso de las TIC, si bien estas diferencias aún son importantes en este tipo de actividades. El problema radica en que no sabemos qué parte de estas diferencias son explicadas por las diferencias existentes entre las características de los trabajadores y cuáles son debidas a la existencia de un trato diferencial del mercado.

Como resulta bien conocido, actualmente en España hombres y mujeres perciben un salario medio diferente, aunque este hecho no se tiene porqué relacionar directamente con la existencia de discriminación, pudiendo estar fundamentado sobre diferentes causas:

a) Ambos colectivos pueden presentar rendimientos salariales diferentes debido a que presentan diferencias en sus características personales y laborales. A pesar de ello, en el entorno español se ha comprobado que existe un pago desigual hacia los hombres que no puede ser explicado únicamente por la existencia de características diferenciales. De la Rica y Ugidos en 1995 desarrollaron uno de los trabajos pioneros en este ámbito, estableciendo que la diferenciación salarial en España se basa en la existencia de un capital humano diferente, aunque sobre todo en la falta de participación laboral femenina, que establece un importante sesgo 
de autoselección de cara al posible cobro de los rendimientos salariales.

b) Por otra parte, las diferencias salariales existentes por género en nuestro país se deben a la difícil y tardía incorporación de la mujer al mercado de trabajo, así como a la segregación sectorial y ocupacional (Aláez y Ullibarri, 1995). Hay que tener en cuenta que, si bien el primer factor ha sido superado en cierta medida, la segregación femenina aún resulta persistente en nuestro país (Iglesias, C. y Llorente, R, 2008).

c) Asimismo, los diferentes rendimientos salariales percibidos por hombres y mujeres también se encuentran influenciados por el establecimiento donde se realiza el empleo, de modo que las mujeres españolas presentan menores rendimientos debido al hecho de estar segregadas hacia puestos de trabajo ubicados en establecimientos asociados al cobro de bajos salarios (Simon, H., 2006). El desarrollo de las TIC suele asociarse a una mayor productividad $\mathrm{y}$, por consiguiente, con un rendimiento salarial superior. La incorporación de la mujer en establecimientos donde se hayan incorporado y se utilicen las TIC podría reducir las diferencias salariales existentes por género.

La forma en que habitualmente se aborda el análisis de la discriminación salarial implica llevar a cabo la denominada descomposición de Oaxaca. Esta metodología cuenta ya con una muy amplia aplicación. En este sentido, y en relación con el objetivo de nuestro trabajo, Plasman y Sissomo (2004), por ejemplo, realizan una interesante y actual revisión de las diferencias salariales existentes por género en diversos países europeos, demostrando que parte de dichas diferencias (menos del 50 por ciento) se explican por la diferente ubicación de hombres y mujeres en el mercado de trabajo (segregación ocupacional y sectorial), si bien lo más importante de este trabajo reside en la constatación de que dichas diferencias varían mucho de unos países a otros en función de las diferencias existentes en capital humano. Ante estos resultados se podría esperar que, en las nuevas generaciones, la discriminación salarial sea menor, dado que las mujeres jóvenes poseen un mayor capital humano y, por consiguiente, pueden evitar con mayor facilidad situaciones laborales relacionadas con la segregación ocupacional y sectorial. Sin embargo, Sami Napari (2009) demuestra para el caso finlandés y en el sector de ocupaciones no manuales de alta cualificación, como durante los primeros años de inserción laboral la segregación salarial no solo no se reduce sino 
que se incrementa espectacularmente ${ }^{3}$. No obstante, la mayor movilidad ocupacional o cambio de status profesional que presentan los hombres durante los primeros años de su carrera profesional podría estar detrás de este hecho.

En definitiva, y a la luz de estas ideas, podemos establecer que si el uso de la TIC mejora la posición laboral de la mujer en cuanto al acceso al empleo y su posible segregación, entonces es posible que la diferencia salarial existente por genero en el desarrollo de las ocupaciones TIC también se reduzca, resultando inferior al resto de ámbitos ocupacionales.

\section{Posición laboral en el empleo TIC}

El empleo TIC se define como la parte del empleo total especialmente relacionado con las Tecnologías de la Información y la Comunicación (OCDE, 2004). Para su medición, la OCDE establece dos diferentes aproximaciones. De acuerdo con la primera de ellas, el empleo TIC se correspondería con el empleo ubicado en los sectores productores de bienes y servicios TIC (sector TIC). Desde la segunda de ellas, el empleo TIC se refiere a todo aquel empleo cuyas ocupaciones laborales muestran una especial relación con las TIC (ocupaciones TIC). La primera definición considera como empleo TIC todo aquel incluido en actividades productoras de bienes y servicios TIC, independientemente de que el contenido de su trabajo se vincule realmente con las TIC y dejando fuera aquel empleo que, relacionado con las TIC, se ubica en sectores no productores de estas tecnologías. Por este motivo, el artículo utiliza la segunda definición para aproximar el empleo TIC; su composición se recoge en el anexo (cuadro A.1).

De acuerdo con los datos de la EPA, en el segundo trimestre de 2007 el empleo TIC, definido en la forma establecida, ascendía en España a un total de 4,311 millones de trabajadores, lo que supone el 21,2 por ciento del conjunto de la población ocupada. El empleo de ocupaciones TIC está compuesto sobre todo por empleados que desarrollan tareas administrativas, con y sin atención al público (22,5 por ciento del total) y encargados de labores comerciales y de gestión administrativa (27,9 por ciento). Con una ponderación algo menor, también destacan las ocupaciones de ingenieros superiores y técnicos, por una parte, y los profesionales de apoyo en operaciones comerciales y comerciales, de otra, ambas con pesos que suponen en torno al 5 por ciento del total (cuadro A.1). El empleo que desarrolla ocupaciones TIC

3 Este entorno también se asemeja a los puestos de trabajo asociados a las TIC con un mayor nivel de cualificación y desarrollo de tareas no manuales de elevada cualificación. 
está ligeramente más feminizado que el resto del empleo. El acceso de la mujer a los puestos TIC resulta difícil, pero una vez logrado alcanza una distribución por género más homogénea (cuadro 1). Además, de las 8,3 millones de mujeres empleadas, el 23,42 por ciento lo hacían en ocupaciones TIC y el 76,58 por ciento lo hacían en ocupaciones no TIC.

Cuadro 1. Distribución por género del empleo en ocupaciones TIC en comparación con el resto. Tantos por ciento. (Fuente: EPA, $2^{\circ}$ trimestre de 2002 y 2007 ).

\begin{tabular}{llll}
\hline & & $\mathbf{2 0 0 2}$ & $\mathbf{2 0 0 7}$ \\
\hline \multirow{2}{*}{ Ocupaciones TIC } & Hombres & $57,63 \%$ & $54,71 \%$ \\
& Mujeres & $42,37 \%$ & $45,29 \%$ \\
\multirow{2}{*}{ Ocupaciones no TIC } & $63,41 \%$ & $59,94 \%$ \\
& Hombres & $36,59 \%$ & $40,06 \%$ \\
\hline
\end{tabular}

En cuanto a las características personales y laborales del empleo que desarrolla ocupaciones relacionadas con las TIC, destaca el hecho de que los trabajadores que desarrollan este tipo de empleo poseen un nivel de estudios notablemente superior. También presentan unas mejores condiciones laborales, con menores contratos temporales y una menor presencia de jornadas a tiempo parcial. Todo ello, tal como se comprueba posteriormente, tiene su repercusión positiva en los rendimientos salariales obtenidos por los trabajadores que desarrollan ocupaciones ligadas con el uso de las TIC (tabla A.2 en el anexo).

Más allá de estos datos iniciales, una primera cuestión interesante consiste en determinar si el empleo TIC presenta también diferencias laborales significativas entre mujeres y hombres. Las características laborales de las mujeres que trabajan en ocupaciones TIC en comparación con las de sus compañeros varones vienen recogidas en las columnas 3 a 5 del cuadro 2. De sus datos se deducen una serie de relevantes pautas de diferenciación entre ambos colectivos:

a) El empleo femenino con ocupaciones TIC es sensiblemente más joven que el masculino, presentando índices de incidencia superiores a la unidad para el intervalo de menor edad.

b) No se observan diferencias significativas desde las perspectivas del estado civil, el nivel de estudios, el sector de actividad y la situación profesional.

c) A pesar de lo cual el empleo femenino en estas ocupaciones presenta tasas de temporalidad y, especialmente, de parcialidad notablemente superiores a la de los hombres. 
Cuadro 2. Características del empleo TIC por género y comparación de las características del empleo femenino en ocupaciones TIC con el ubicado en el resto de ocupaciones. Tantos por ciento e índices de incidencia. (Fuente: EPA, $2^{\circ}$ trimestre de 2007).

\begin{tabular}{|c|c|c|c|c|c|c|c|}
\hline & \multirow[b]{2}{*}{ Características } & \multicolumn{3}{|c|}{ Distribución por género del empleo TIC } & \multicolumn{3}{|c|}{$\begin{array}{l}\text { Distribución ocupacional } \\
\text { del empleo femenino }\end{array}$} \\
\hline & & Mujeres & Hombres & $\begin{array}{c}\text { Incidencia } \\
\text { (Mujeres } \\
\text { sobre } \\
\text { Hombres) }\end{array}$ & $\begin{array}{c}\text { Ocup. } \\
\text { TIC }\end{array}$ & Resto & $\begin{array}{c}\text { Incidencia } \\
\text { (Ocup. TIC } \\
\text { sobre } \\
\text { Resto) }\end{array}$ \\
\hline \multirow{3}{*}{ Edad } & Jóvenes & 48,2 & 38,0 & 1,3 & 48,2 & 43,5 & 1,11 \\
\hline & Intermedios & 45,1 & 48,8 & 0,9 & 45,1 & 46,3 & 0,97 \\
\hline & Maduros & 6,6 & 13,1 & 0,5 & 6,6 & 10,2 & 0,65 \\
\hline \multirow{2}{*}{$\begin{array}{l}\text { Estado } \\
\text { civil }\end{array}$} & Soltero/a & 37,5 & 34,3 & 1,1 & 37,5 & 35,0 & 1,07 \\
\hline & No soltero/a & 62,5 & 65,7 & 1,0 & 62,5 & 65,0 & 0,96 \\
\hline \multirow{3}{*}{ Estudios } & Educación primaria & 1,9 & 3,3 & 0,6 & 1,9 & 15,1 & 0,12 \\
\hline & Educación secundaria & 39,6 & 37,0 & 1,1 & 39,6 & 52,9 & 0,75 \\
\hline & Educación superior & 58,5 & 59,7 & 1,0 & 58,5 & 32,0 & 1,83 \\
\hline \multirow{2}{*}{$\begin{array}{l}\text { Sector de } \\
\text { actividad }\end{array}$} & Actividades TIC & 5,9 & 13,0 & 0,5 & 5,9 & 0,9 & 6,91 \\
\hline & Resto de actividades & 94,1 & 87,0 & 1,1 & 94,1 & 99,1 & 0,95 \\
\hline \multirow{2}{*}{$\begin{array}{l}\text { Situación } \\
\text { prof. }\end{array}$} & Asalariados/as & 88,8 & 80,4 & 1,1 & 88,8 & 85,8 & 1,04 \\
\hline & No asalariados/as & 11,2 & 19,6 & 0,6 & 11,2 & 14,2 & 0,78 \\
\hline \multirow{2}{*}{$\begin{array}{l}\text { Tipo de } \\
\text { contrato }\end{array}$} & Indefinido & 79,9 & 85,6 & 0,9 & 79,9 & 62,6 & 1,28 \\
\hline & Temporal & 20,1 & 14,4 & 1,4 & 20,1 & 37,4 & 0,54 \\
\hline \multirow{2}{*}{$\begin{array}{l}\text { Tipo de } \\
\text { jornada }\end{array}$} & Completa & 85,5 & 96,8 & 0,9 & 85,5 & 74,4 & 1,15 \\
\hline & Parcial & 14,5 & 3,2 & 4,5 & 14,5 & 25,6 & 0,56 \\
\hline
\end{tabular}

Los datos apuntan, por tanto, que aunque se observan características similares para hombres y mujeres desde la mayoría de las características, el empleo femenino relacionado con las ocupaciones TIC se asocia con peores condiciones laborales (temporalidad y parcialidad).

Otra cuestión relevante consiste en determinar en qué medida las mujeres ocupadas en el empleo TIC mejoran o no las condiciones laborales de sus compañeras ubicadas en el resto del empleo. En este sentido, y de acuerdo con los datos de las columnas 6 a 8 del cuadro 2, las pautas que se deducen se resumen en los siguientes hechos:

a) Las mujeres vinculadas con las ocupaciones TIC son más jóvenes que las mujeres que desempeñan otro tipo de ocupaciones. 
b) Por lo que la presencia de solteras resulta ligeramente más frecuente entre las mujeres empleadas en ocupaciones TIC.

c) Acentúan muy notablemente su presencia sobre los estudios de grado superior.

d) Se vinculan preferentemente con el sector productor de bienes y servicios TIC.

e) La tasa de asalarización resulta ligeramente más elevada.

f) Y, sobre todo, limitan en buena medida la presencia de empleo a tiempo parcial y la contratación temporal.

Se trata, por tanto, de un colectivo de mujeres jóvenes, formadas y que se asocian con mejores condiciones laborales de lo que es propio para el conjunto de las mujeres en el sistema productivo. Todo ello, apunta a que la mujer que desarrolle ocupaciones relacionadas con las TIC presente una posición salarial ventajosa respecto al resto de mujeres.

\section{Discriminación salarial y empleo TIC}

A partir de los análisis desarrollados previamente, nuestro objetivo ahora es doble. Por una parte, conocer si en el empleo TIC existe discriminación salarial. De otra, determinar cuál es su intensidad en comparación con el resto de ocupaciones no asociadas a las TIC. La hipótesis fundamental sería que las nuevas Tecnologías de la Información y la Comunicación atesoran un elevado potencial para mejorar la situación laboral de la mujer, reduciendo los niveles de desigualdad laboral existentes por género. Sin embargo, es posible que existan situaciones que dificulten estas mejoras, de manera que la reducción de la discriminación se vea moderada. Por ejemplo, y tal como señala la literatura feminista, la situación laboral de la mujer puede ser consecuencia de un proceso de socialización sesgado por género, donde las oportunidades laborales de las mujeres estarían condicionadas por la división doméstica del trabajo y las políticas públicas aplicadas en este sentido (Figart, Mutari y Power, 2002).

Para analizar estas cuestiones, estudiaremos cómo son los rendimientos salariales por género, comparando la situación existente en el empleo relacionado con ocupaciones TIC y en el resto (empleo no TIC). Desde un punto de vista metodológico esto implica proceder a la estimación de una serie de ecuaciones de ingreso o rendimientos salariales, identificando qué parte de la remuneración se puede atribuir a las características presentadas por los trabajadores o por el puesto de trabajo (educación, experiencia laboral y variables personales). Mediante 
la aplicación de la descomposición de Oaxaca-Blinder a los datos salariales aportados por la ECV-2008 podremos determinar qué parte de las diferencias salariales existentes entre las ocupaciones TIC y no TIC, y posteriormente, por género, no se corresponden con diferencias observadas en las características personales y profesionales, sino que se deben identificar con la existencia de situaciones de discriminación laboral o tratamiento diferencial.

Aunque los datos aportados por la ECV resultan adecuados a nuestros propósitos, presentan el inconveniente de la escasa desagregación que presenta la información relativa a las ocupaciones laborales de los trabajadores, alcanzando sólo un detalle de dos dígitos. Esto implica que debemos de redefinir el concepto de empleo relacionado con ocupaciones TIC desde los tres dígitos utilizados por la definición de la OCDE hasta los dos dígitos aportados por la ECV. El resultado de este ajuste se recoge el anexo (cuadro A.1).

\subsection{La remuneración salarial en el empleo TIC frente al resto.}

El cuadro 3 resume la situación salarial existente en 2008 de acuerdo con los datos de la ECV-2008. De acuerdo a estos datos, los trabajadores que desarrollan ocupaciones TIC a tiempo completo presentaban en 2008 un salario bruto mensual medio de 2.174 euros, mientras que el resto de trabajadores presentan un salario bruto medio en torno a 1.726,2 euros. Esto supone una mejora salarial de 447,8 euros mensuales brutos para aquellos trabajadores que realizaban ocupaciones laborales asociadas a las TIC (lo que supone aproximadamente un 25 por ciento más).

Por otra parte, en las ocupaciones TIC, el salario medio bruto anual femenino es de 1.968,3 euros mientras que el masculino es de 2.423,3 euros brutos, siendo de esta forma la diferencia salarial existente entre hombres y mujeres de 455 euros brutos mensuales (un 23,1 por ciento más). Aunque la mujer en las ocupaciones TIC alcanza sueldos superiores al resto de mujeres empleadas en otras ocupaciones, su salario aún se encuentra lejos de los rendimientos salariales obtenidos por los hombres ${ }^{4}$.

\footnotetext{
${ }^{4}$ Diaz-Chao (2008) obtiene que esta diferencia es de un 34 por ciento al considerar el salario por hora de trabajadores vinculados a empresas que desarrollan actividades pertenecientes a la industria del conocimiento frente al resto de actividades productivas con datos de la Encuesta Anual de Estructura Salarial 2002.
} 


\section{Cuadro 3. Situación salarial por género en las ocupaciones TIC y} no TIC. (Fuente: ECV-2008. INE).

\begin{tabular}{lccc}
\hline Salario bruto mensual por trabajador (euros) & Cuantía & $\begin{array}{c}\text { Dif. bruta con el } \\
\text { asalariado a TC }\end{array}$ & $\begin{array}{c}\text { Desviación } \\
\text { Estándar }\end{array}$ \\
\hline $\begin{array}{l}\text { Salario medio por asalariado (TODOS): } \\
\text { Salario medio por trabajador asalariado a tiempo } \\
\text { completo (TC): }\end{array}$ & $1.700,6$ & $-127,2$ & $1.001,9$ \\
$\begin{array}{l}\text { Salario medio por trabajador asalariado a tiempo } \\
\text { completo (HOMBRE-TC): }\end{array}$ & $1.827,8$ & - & 986,1 \\
$\begin{array}{l}\text { Salario medio por trabajador asalariado a tiempo } \\
\text { completo (MUJER-TC): }\end{array}$ & $1.956,3$ & 128,5 & $1.055,6$ \\
$\begin{array}{l}\text { Salario medio por trabajador asalariado a tiempo } \\
\text { completo (OCUP-NO TIC-TC): }\end{array}$ & $1.726,2$ & $-197,4$ & 830,8 \\
$\begin{array}{l}\text { Salario medio por trabajador asalariado a tiempo } \\
\text { completo (OCUP- TIC-TC): }\end{array}$ & $2.174,0$ & $-101,7$ & 932,8 \\
$\begin{array}{l}\text { Salario medio por trabajador asalariado a tiempo } \\
\text { completo (OCUP-TIC-HOMBRE-TC): }\end{array}$ & $2.423,3$ & 346,2 & $1.079,7$ \\
$\begin{array}{l}\text { Salario medio por trabajador asalariado a tiempo } \\
\text { completo (OCUP-TIC-MUJER-TC): }\end{array}$ & $1.968,3$ & 595,5 & $1.282,0$ \\
$\begin{array}{l}\text { Salario medio por trabajador asalariado a tiempo } \\
\text { completo (OCUP- NO TIC-HOMBRE-TC): }\end{array}$ & $1.861,0$ & 140,5 & 824,0 \\
$\begin{array}{l}\text { Salario medio por trabajador asalariado a tiempo } \\
\text { completo (OCUP-NO TIC-MUJER-TC): }\end{array}$ & $1.474,8$ & 33,2 & 976,3 \\
\hline
\end{tabular}

Por último, señalar cómo la diferencia salarial por género dentro de las TIC resulta superior a la existente en el resto de ocupaciones. El gap salarial por género en las TIC es de 455 euros mientras que el mismo dato para el resto de ocupaciones es de 325,8 euros. Asimismo, la dispersión salarial a la que se enfrentan las mujeres dentro de las TIC es elevada (con una desviación estándar de 824 euros) pero no resulta tan elevada como la de los hombres (desviación estándar de 1.282 euros).

Estos datos podrían llevarnos a pensar que la mujer se encuentra salarialmente más discriminada en las ocupaciones TIC. Sin embargo, hay que tener en cuenta que parte de estas diferencias se deben a que hombres y mujeres presentan características diferentes a remunerar. Para controlar por dicho sesgo, a continuación se desarrolla una serie de ecuaciones salariales que estiman el pago a los asalariados a tiempo completo teniendo en cuenta sus características personales y profesionales, como base sobre la que estimar posteriormente el gap salarial existente en diferentes entornos. Las ecuaciones salariales estimadas son las estándar y se establecen de acuerdo a la siguiente formulación:

$$
\ln W=\alpha_{0}+\alpha_{1} X_{i}+\mu
$$


Donde $\ln W$ es el logaritmo neperiano del salario bruto mensual, $X_{i}$ es un vector de variables sobre las características personales y profesionales por las cuales cada individuo es remunerado, y $\mu$ es el término de error.

La decisión de considerar el salario bruto mensual como variable a explicar se fundamenta en el hecho de que creemos que las necesidades de conciliación laboral y familiar se constituyen en una situación de la máxima relevancia cuando estudiamos comportamientos laborales femeninos, siendo mucho mejor incorporada mediante el salario mensual que mediante su alternativa, el salario hora. Además, la variable salario/hora no permite considerar en el análisis el hecho de que mujeres y hombres puedan presentar diferencias significativas en la duración de su jornada de trabajo.

El rendimiento salarial se ha estimado considerando como variables explicativas el género, el estado civil (que muestra si la persona se encuentra casada frente al resto de estados civiles posibles), la nacionalidad (que divide a la población entre nacionales y extranjeros), el nivel de estudios (que diferencia entre 5 niveles: estudios primarios, estudios de secundaria de primera etapa, estudios de secundaria de segunda etapa, estudios de Formación Profesional e inserción laboral, y estudios superiores), la experiencia potencial (que mide el número de años desde que se finalizaron los estudios), la experiencia potencial al cuadrado $^{5}$, las horas de trabajo (considerando sólo las correspondientes a los asalariados a tiempo completo), el tamaño de la empresa (diferenciando en cuatro niveles: establecimientos de 1 a 10 trabajadores, de 11 a 19, de 20 a 49 y 50 o más) y si el individuo presenta un contrato temporal ${ }^{6}$.

La elección de considerar sólo el trabajo a tiempo completo encuentra a nuestro juicio su justificación sobre varios argumentos. En primer lugar hemos de recordar que trabajamos con salarios brutos mensuales, lo que supone que existiría una gran diferencia bruta entre lo

\footnotetext{
${ }^{5}$ La inclusión de la experiencia potencial al cuadrado intenta expresar el hecho de que la experiencia vital y laboral no se acumula de manera lineal a lo largo de la vida del individuo. También fueron desarrollados modelos considerando la edad como variable representativa de la experiencia vital; sin embargo, sus estimaciones presentaron una significatividad levemente menor. Además, dado que edad y experiencia se revelaron como altamente correlacionadas, finalmente se optó por incluir solamente en los modelos la experiencia laboral y su cuadrado.

${ }^{6}$ No se considera como variable explicativa el sector de actividad de colocación debido a su elevada correlación con las ocupaciones laborales, variable que se utiliza posteriormente para definir el empleo TIC y no TIC y, por tanto, fundamento de la selección desarrollada en los diferentes modelos analizados.
} 
que cobran los trabajadores a tiempo completo y los que lo hacen a tiempo parcial. La inclusión en el modelo de trabajadores a tiempo parcial puede conllevar un cierto sesgo o ruido. Asimismo, aunque la ECV-2008 cuenta con muestra suficiente para el estudio de los trabajadores a tiempo parcial, no tiene suficientes datos sobre mujeres ocupadas en las TIC que trabajen a tiempo parcial, escasez que se debe más a la realidad económica de nuestro país que a la propia encuesta. Por último, trabajar con tiempo completo establece una comparación más homogénea entre hombres y mujeres, dado que el tiempo parcial incide preferentemente sobre las mujeres.

En las ecuaciones salariales suele ser habitual controlar las estimaciones de acuerdo con el sesgo de selección de Heckman, de modo que se incluye en la ecuación un nuevo componente que pondera las estimaciones de acuerdo con la probabilidad de estar ocupado (conocido como el sesgo de selección) ${ }^{7}$. Sin embargo, en nuestro caso esto puede ser contraproducente, dado que deseamos analizar las diferencias salariales entre diferentes grupos de forma completa, sin ser corregidas por dicha selección. Téngase en cuenta que, al incluir el sesgo de selección, los porcentajes de discriminación, al menos por sexo, suelen reducirse (Hernandez y Méndez, 2005) dado que se está estimando el gap salarial respecto a una situación hipotética corregida para que los diferentes grupos de contraste tengan una misma probabilidad de participación. Por otra parte, al incluir un término de corrección en la estimación de las diferencias salariales se puede distorsionar el resto de parámetros obteniendo un resultado incierto respecto a la discriminación absoluta ${ }^{8}$. Asimismo, dado que nuestras estimaciones se concentran en la población ocupada, concretamente en los asalariados que trabajan a tiempo completo dentro y fuera de las TIC, diferenciando entre hombre y mujeres, por lo cual no resulta necesario analizar el proceso de selección laboral hacia este tipo de ocupaciones, sino la propia situación de discriminación existente dentro de las $\mathrm{TIC}^{9}$.

\footnotetext{
7 En este caso la estimación sería la siguiente: $\ln W_{i}=\alpha_{0}+\alpha_{1} X_{i}+\alpha_{2} Y^{i}+\mu$ donde Yi sería el término de corrección conocido como la inversa del ratio de Mills que contempla en la estimación la posible aleatoriedad de formar parte del colectivo de asalariados dentro de cada uno de los diferentes grupos considerados.

8 Por ejemplo, Dolado et al (2002) utilizan estimaciones del gap salarial por género sin corregir por el sesgo de selección para conocer el valor real de la discriminación. En este trabajo la estimación del gap salarial se incluye en una serie de modelos sobre la diferente segregación ocupacional existente en EEUU y Europa de modo que interesa contar con estimaciones brutas de dicha diferencia salarial.

9 Esta decisión también se fundamenta en el hecho de intentar trabajar con los datos más homogéneos posibles en relación al salario. Aunque la ECV cuenta con datos sobre
} 
Cuadro 4. Ecuaciones de salarios para ocupaciones TIC y no TIC. (Fuente: elaboración propia sobre datos de la ECV-2008, INE) ${ }^{10}$.

\begin{tabular}{|c|c|c|c|c|c|c|}
\hline \multirow[b]{2}{*}{$\ln W$} & \multicolumn{3}{|c|}{ Ocupaciones no TIC } & \multicolumn{3}{|c|}{ Ocupaciones TIC } \\
\hline & Coef. & $\begin{array}{c}\text { Error } \\
\text { estándar }\end{array}$ & $\mathbf{P}>\mathbf{z}$ & Coef. & $\begin{array}{c}\text { Error } \\
\text { estándar }\end{array}$ & $\mathbf{P}>\mathbf{z}$ \\
\hline Mujer & $-0,223$ & 0,011 & $0,000 * *$ & $-0,155$ & 0,019 & $0,000^{* *}$ \\
\hline Casado & 0,132 & 0,012 & $0,000^{* * *}$ & 0,114 & 0,022 & $0,000 * *$ \\
\hline Español & 0,120 & 0,018 & $0,000 * *$ & 0,111 & 0,064 & $0,083^{*}$ \\
\hline Estudios de secundaria 1ra. etapa & 0,067 & 0,014 & $0,000 * *$ & 0,194 & 0,077 & $0,012^{* *}$ \\
\hline Estudios de secundaria 2da. etapa & 0,209 & 0,016 & $0,000 * *$ & 0,321 & 0,073 & $0,000 * *$ \\
\hline FP e inserción laboral & 0,260 & 0,039 & $0,000 * *$ & 0,365 & 0,162 & $0,025^{* *}$ \\
\hline Estudios superiores & 0,471 & 0,018 & $0,000 * *$ & 0,627 & 0,073 & $0,000 * *$ \\
\hline Experiencia potencial & 0,012 & 0,002 & $0,000 * *$ & 0,020 & 0,003 & $0,000^{* *}$ \\
\hline Experiencia potencial ${ }^{2}$ & 0,000 & 0,000 & $0,000 * *$ & 0,000 & 0,000 & $0,004 * *$ \\
\hline Horas de trabajo & 0,009 & 0,001 & $0,000 * *$ & 0,006 & 0,002 & $0,001 * *$ \\
\hline Empresa de 1 a 10 trabajadores & $-0,057$ & 0,014 & $0,000 * *$ & $-0,080$ & 0,029 & $0,006^{* * *}$ \\
\hline Empresa de 20 a 49 trabajadores & 0,058 & 0,018 & $0,001^{* *}$ & 0,046 & 0,029 & 0,111 \\
\hline Empresa de 50 o más trabajadores & 0,169 & 0,014 & $0,000^{* *}$ & 0,109 & 0,026 & $0,000 * *$ \\
\hline Temporal & $-0,133$ & 0,012 & $0,000^{* *}$ & $-0,178$ & 0,027 & $0,000 * *$ \\
\hline Constante & 6,477 & 0,053 & $0,000 * *$ & 6,492 & 0,123 & $0,000^{* * *}$ \\
\hline $\mathrm{N}^{0}$ de observaciones no censuradas & \multicolumn{3}{|c|}{7.779} & \multicolumn{3}{|c|}{2.171} \\
\hline $\mathrm{R}^{2}$ & \multicolumn{3}{|c|}{0,400} & \multicolumn{3}{|c|}{0,381} \\
\hline Sig. & \multicolumn{3}{|c|}{0,000} & \multicolumn{3}{|c|}{0,000} \\
\hline
\end{tabular}

* Significativo bajo una probabilidad del $90 \%$

Los rendimientos salariales se han estimado primero para los trabajadores que desarrollan tareas TIC y posteriormente para el resto de trabajadores. A continuación, se vuelve a ejecutar esta descomposición considerando la diferencia entre hombres y mujeres, ahora solo en relación con las tareas TIC. En el anexo se incluyen las diferencias salariales existentes por género al desarrollar tareas no relacionadas con las TIC.

De acuerdo con este orden, en el cuadro 4 se recogen las estimaciones de los rendimientos salariales para el empleo TIC y no TIC. Sus resultados establecen las siguientes pautas generales:

horas trabajadas, los datos relativos a los salarios suelen ser mensuales, por ello, una estimación del salario por hora puede contener una elevada heterogeneidad sobre todo si se contempla el empleo parcial.

10 Como ejercicio inicial, en primer lugar se estimó la influencia aislada del género en las ganancias salariales para toda la muestra, encontrando que en términos brutos las mujeres ganan un 32 por ciento menos. Sin embargo, al diferenciar esta estimación para las tareas TIC, encontramos que las mujeres dentro de las TIC ganan en términos brutos un 19 por ciento menos. 
a) Estar casado favorece la percepción de rendimientos salariales superiores, pero sobre todo en el caso de desarrollar ocupaciones no relacionadas con las TIC.

b) Ser español incrementa el rendimiento salarial, resultando esta pauta levemente superior en el caso de desarrollar ocupaciones fuera de las TIC.

c) Los niveles de estudios presentan coeficientes significativos, y al ser todos positivos quieren decir que el salario aumenta a medida que se incrementa la formación siendo esta influencia más intensa en las TIC que fuera de ellas.

d) La experiencia laboral muestra un coeficiente positivo aunque de escasa cuantía indicando simplemente que la permanencia en el mercado de trabajo favorece la empleabilidad.

e) Las horas de trabajo también muestran una influencia positiva, aunque de escasa cuantía. No obstante, debe tenerse en cuenta que nuestras estimaciones se realizan sobre los trabajadores con una jornada laboral a tiempo completo y, por tanto, la variabilidad del tiempo de trabajo debe resultar reducida.

f) En las empresas de más de 50 trabajadores es donde se alcanzan mayores rendimientos salariales.

g) Y, por último, tener un contrato temporal reduce el rendimiento salarial, siendo esta disminución más o menos similar al desarrollar ocupaciones TIC o no TIC.

Las ecuaciones anteriores establecen los determinantes de los rendimientos salariales obtenidos en uno y otro ámbito ocupacional pero no explican a qué se debe el gap salarial existente entre las ocupaciones TIC y no TIC. Para resolver esta cuestión hemos llevado a cabo una descomposición del diferencial salarial. Siguiendo la metodología de Oaxaca (1973) y Blinder (1973) el gap salarial existente entre dos grupos laborales diferentes, una vez estimadas las ecuaciones salariales previas, puede descomponerse en varios componentes a través de una simple diferenciación matemática. Siguiendo a Neumark (1988) y Oaxaca y Ransom $(1994,1999)$ una posible diferenciación sería la siguiente:

$$
\begin{aligned}
& \bar{Y}_{i}-\bar{Y}_{j}=\left(\bar{X}_{i}^{\prime}-\bar{X}_{j}^{\prime}\right) \hat{\beta}_{j}+\bar{X}_{j}^{\prime}\left(\hat{\beta}_{i}-\hat{\beta}_{j}\right)+\left(\bar{X}_{i}^{\prime}-\bar{X}_{j}^{\prime}\right)\left(\hat{\beta}_{i}-\hat{\beta}_{j}\right) \\
& \bar{Y}_{i}-\bar{Y}_{j}=\left(\bar{X}_{i}^{\prime}-\bar{X}_{j}^{\prime}\right) \hat{\beta}^{*}+\bar{X}_{i}^{\prime}\left(\hat{\beta}_{i}-\hat{\beta}^{*}\right)+\bar{X}_{j}^{\prime}\left(\hat{\beta}^{*}-\hat{\beta}_{j}\right)
\end{aligned}
$$

De este modo, de nuevo, puede diferenciarse entre: 
a) $\left(\bar{X}_{i}^{\prime}-\bar{X}_{j}^{\prime}\right) \hat{\beta}$ * es el componente "explicado" que se debe a las diferentes dotaciones a remunerar que presentan los grupos de contraste. En este caso $\hat{\beta}^{*}$ es una estimación del coeficiente medio de "no discriminación" utilizando la estimación de salarios incluidos en la regresión previa.

b) $\bar{X}_{i}^{\prime}\left(\hat{\beta}_{i}-\hat{\beta}^{*}\right)$ es el componte que estima el "favoritismo" existente por el primer grupo o la diferencia salarial que recibiría el segundo grupo si fuera tratado como el primero.

c) $\bar{X}_{j}^{\prime}\left(\hat{\beta}^{*}-\hat{\beta}_{j}\right)$ es el componente que recoge la interacción de los efectos previos y, por tanto, directamente atribuible a la parte no explicada por la estimación. Se corresponde con la diferencia salarial conjunta que existiría si el primer grupo tuviera los rendimientos salariales del segundo, y las características del segundo grupo tuvieran los mismos rendimientos salariales que son atribuidos al primer grupo. En definitiva, es la diferencia salarial atribuible no a la discriminación del segundo grupo respecto del primero (componente previo) sino a la discriminación de ambos grupos, es decir, la interacción del mercado o la estructura por género establecida en el pago de salarios.

Los dos últimos componentes sumarían el gap salarial "no explicado" que podría ser equiparado con la discriminación salarial o el diferente tratamiento salarial sin justificación. Los resultados de esta aproximación econométrica han sido recogidos en el siguiente cuadro número 5. Puede observarse que todos los componentes obtienen coeficientes significativos, determinando que la descomposición en tres términos se encuentra adecuadamente establecida ${ }^{11}$.

De acuerdo con estas estimaciones, la diferencia salarial existente entre los trabajadores TIC y no TIC se explicaría en un 48,3 por ciento por las mejores dotaciones que presentan los trabajadores de las TIC, en un 34,2 por ciento por el tratamiento preferencial que tienen estos trabajadores, y un 17,5 por ciento se relacionaría con la interacción de ambos efectos. Aunando los dos componentes finales se puede establecer que un 51,7 por ciento del gap salarial no puede explicarse de acuerdo a las características personales y profesionales establecidas.

\footnotetext{
$11 \mathrm{El}$ efecto interacción es significativo y no se puede reducir la descomposición tan solo a la valoración del componente explicado y no explicado, o como se desarrolla en la estimación tradicional de Oaxaca-Blinder.
} 
Cuadro 5. Descomposición de Oaxaca sobre el diferencial de salarios existente entre las ocupaciones TIC y no TIC. (Fuente: elaboración propia sobre datos de la ECV-2008, INE).

\begin{tabular}{|c|c|c|c|c|c|}
\hline \multirow[b]{2}{*}{ Diferencial } & \multicolumn{4}{|c|}{ Valores del LnW } & \multirow{2}{*}{$\begin{array}{c}\text { Valores } \\
\text { monetarios } \\
\text { (euros) }\end{array}$} \\
\hline & Coef. & Std. Err. & $\mathbf{P}>\mathbf{z}$ & $\%$ & \\
\hline Predicción del lnW en TIC & 7,587 & 0,012 & $0,000 * *$ & & $1.971,5$ \\
\hline Predicción del $\ln W$ en no TIC & 7,349 & 0,007 & $0,000 * *$ & & $1.554,1$ \\
\hline Diferencia de $\ln W$ & 0,238 & 0,013 & $0,000 * *$ & & 417,4 \\
\hline \multicolumn{6}{|l|}{ Descomposición } \\
\hline Dotaciones & 0,115 & 0,010 & $0,000^{* *}$ & 48,3 & 201,5 \\
\hline Coeficientes & 0,081 & 0,018 & $0,000 * *$ & 34,2 & 142,8 \\
\hline Interacción (variables y coeficientes) & 0,042 & 0,016 & $0,008^{* *}$ & 17,5 & 73,2 \\
\hline
\end{tabular}

En términos monetarios, los componentes previos se traducen en que los trabajadores de las TIC obtienen como remuneración a sus características personales y laborales 201,5 euros mensuales más que el resto de trabajadores, obteniendo en media 142,8 euros mensuales más solo por realizar ocupaciones TIC o por recibir un mejor tratamiento, y la interacción del mercado laboral hace que además se sumen en media 73,2 euros más. Por tanto, en las ocupaciones TIC existe un tratamiento diferencial de carácter positivo que según estas estimaciones se cifra en 216 euros mensuales cuyo pago no puede atribuirse a la remuneración de las características personales o laborales de los trabajadores y que, tal vez, puede asociarse a la existencia de un trato discriminatorio o diferencial.

\subsection{Los rendimientos salariales que obtienen las mujeres en las ocupaciones TIC}

Queda demostrado que en las ocupaciones TIC los rendimientos salariales son superiores. La siguiente pregunta debe ser: ¿Cuál es la posición de hombre y mujeres dentro de las TIC? y ¿cómo son los rendimientos salariales de hombres y mujeres al desarrollar ocupaciones TIC? Siguiendo la metodología establecida en el apartado anterior, en primer lugar, se desarrollan una serie de ecuaciones salariales para hombres y mujeres que realizan ocupaciones relacionadas con las TIC, para posteriormente, a través del uso de la descomposición salarial, establecer a qué se deben las diferencias observadas. Asimismo, en el anexo se desarrolla la misma metodología para las ocupaciones no TIC utilizadas como referencia de comparación (cuadro A.4 y A.5). 
De acuerdo con las ecuaciones de salarios para los empleados TIC (cuadro 6), las diferencias claves que se observan entre la determinación del salario de hombres y mujeres en ocupaciones TIC se concretan en que el rendimiento salarial de la mujer, al contrario que los hombres, no se encuentra tal altamente influenciado y de forma positiva por la condición de ser casado. Además, ser español es algo determinante de los rendimientos salariales de la mujer, sin embargo, en el caso de los hombre son resulta significativo. La escasa presencia de mujeres extranjeras desarrollando este tipo de ocupaciones puede ser la causa de este resultado. El rendimiento salarial de las mujeres en las ocupaciones TIC se asocia en gran medida con su mayor formación. Tener estudios superiores incrementa los salarios mensuales de los hombres y las mujeres en las ocupaciones TIC, sin bien, este hecho es más intenso en el caso de los hombres las diferencias en términos educativos son más acusadas en el caso femenino. La experiencia laboral incrementa levemente y más o menos del mismo modo la empleabilidad dentro de las TIC entre los hombre que entre las mujeres. Por último, tener un contrato temporal reduce la remuneración sobre todo en el caso de las mujeres.

Cuadro 6. Ecuaciones de salarios para los hombres y mujeres que desarrollan una ocupación TIC en su puesto de trabajo. (Fuente: elaboración propia sobre datos de la ECV-2008, INE).

\begin{tabular}{|c|c|c|c|c|c|c|}
\hline \multirow[b]{2}{*}{$\ln W$} & \multicolumn{3}{|c|}{ Hombres en ocupaciones TIC } & \multicolumn{3}{|c|}{ Mujeres en ocupaciones TIC } \\
\hline & Coef. & $\begin{array}{c}\text { Error } \\
\text { estándar }\end{array}$ & $\mathbf{P}>\mathbf{z}$ & Coef. & $\begin{array}{c}\text { Error } \\
\text { estándar }\end{array}$ & $\mathbf{P}>\mathbf{z}$ \\
\hline Casado & 0,188 & 0,035 & $0,000 * *$ & 0,059 & 0,028 & $0,035^{* *}$ \\
\hline Español & 0,070 & 0,095 & 0,461 & 0,134 & 0,081 & $0,096^{*}$ \\
\hline Estudios de secundaria 1ra. etapa & 0,236 & 0,103 & $0,023^{* *}$ & 0,129 & 0,106 & 0,224 \\
\hline Estudios de secundaria 2da. etapa & 0,308 & 0,099 & $0,002^{* *}$ & 0,307 & 0,102 & $0,003^{* *}$ \\
\hline FP e inserción laboral & 0,469 & 0,225 & $0,038^{* *}$ & 0,146 & 0,124 & 0,242 \\
\hline Estudios superiores & 0,632 & 0,098 & $0,000 * *$ & 0,601 & 0,100 & $0,000^{* * *}$ \\
\hline Experiencia potencial & 0,021 & 0,006 & $0,000^{* *}$ & 0,019 & 0,004 & $0,000^{* * *}$ \\
\hline Experiencia potencial $^{2}$ & 0,000 & 0,000 & $0,023^{* *}$ & 0,000 & 0,000 & $0,067^{*}$ \\
\hline Horas de trabajo & 0,008 & 0,002 & $0,002^{* *}$ & 0,003 & 0,003 & 0,345 \\
\hline Empresa de 1 a 10 trabajadores & $-0,015$ & 0,044 & 0,725 & $-0,121$ & 0,039 & $0,002^{* * *}$ \\
\hline Empresa de 20 a 49 trabajadores & 0,084 & 0,045 & $0,063^{*}$ & 0,017 & 0,037 & 0,653 \\
\hline Empresa de 50 o más trabajadores & 0,162 & 0,040 & $0,000 * *$ & 0,074 & 0,034 & $0,030^{* * *}$ \\
\hline Temporal & $-0,134$ & 0,052 & $0,010 * *$ & $-0,200$ & 0,030 & $0,000^{* * *}$ \\
\hline Constante & 6,367 & 0,165 & $0,000 * *$ & 6,523 & 0,190 & $0,000^{* * *}$ \\
\hline $\mathrm{N}^{\circ}$ de observaciones no censuradas & & 887 & & & 1.284 & \\
\hline $\mathrm{R}^{2}$ & & 0,342 & & & 0,370 & \\
\hline Sig. & & 0,000 & & & 0,000 & \\
\hline
\end{tabular}


A partir de estas estimaciones salariales, se ha calculado la descomposición de Oaxaca sobre el diferencial salarial existente entre hombres y mujeres empleados en las TIC (cuadro 7). Los resultados obtenidos muestran que un 19 por ciento de las diferencias por género observadas dentro las ocupaciones TIC se debe a la diferente dotación que presentan hombres y mujeres; un 77 por ciento se debe al trato diferencial entre hombres y mujeres. Desafortunadamente el componente de la interacción por sí solo no resulta significativo y poco se puede establecer al respecto. No obstante, a nivel global supone que un 81 por ciento del diferente pago que reciben las mujeres respecto a los hombres al desarrollar ocupaciones TIC no puede ser explicado y/o puede asociarse a un trato discriminatorio.

En términos monetarios (véase cuadro 7) los hombres ganan, de acuerdo a las estimaciones, 397 euros más que las mujeres desarrollando tareas TIC, de los cuales 75,4 euros brutos se deben a la existencia de diferencias en su dotación. Pero, los hombres que desarrollan ocupaciones TIC cobran 321,6 euros brutos más al mes en media que las mujeres en la misma situación que no pueden ser explicados por la existencia de diferencias productivas (suma de los dos últimos efectos).

Cuadro 7. Descomposición de Oaxaca sobre el diferencial de salarios existente entre hombres y mujeres que trabajan en ocupaciones TIC. (Fuente: elaboración propia sobre datos de la ECV-2008, INE).

\begin{tabular}{lccccc}
\hline & \multicolumn{3}{c}{ Descomposición del LnW } & $\begin{array}{c}\text { Valores monetarios } \\
\text { (euros) }\end{array}$ \\
\hline Diferencial & Coef. & Std. Err. & $\mathbf{P}>\mathbf{z}$ & $\%$ & \\
Predicción del lnW en TIC-Hombres & 7,697 & 0,018 & $0,000^{* *}$ & & $2.201,8$ \\
Predicción del lnW en TIC-Mujeres & 7,498 & 0,015 & $0,000^{* *}$ & & $1.804,8$ \\
Diferencia de lnW & 0,199 & 0,023 & $0,000^{* *}$ & & 397,0 \\
\hline Descomposición & & & & & \\
\hline Dotaciones & 0,038 & 0,015 & $0,012^{* *}$ & 19,0 & 75,4 \\
Coeficientes & 0,153 & 0,019 & $0,000^{* *}$ & 77,0 & 305,8 \\
Interacción (variables y coeficientes) & 0,008 & 0,011 & 0,455 & 4,0 & 15,8 \\
\hline ** Significativo bajo una probabilidad del $95 \%$. & & & & \\
* Significativo bajo una probabilidad del $90 \%$. & & & &
\end{tabular}

En el anexo (cuadro A.4 y A.5) se recogen todos estos cálculos para las ocupaciones no TIC, demostrándose que en este caso el gap salarial a favor de los hombres se cifra en 350,2 euros de media, 46,8 euros menos que en las TIC, de los cuales 332,0 no vienen explicados por diferencias en dotaciones ${ }^{12}$. Por consiguiente, aunque las mujeres dentro de las

\footnotetext{
${ }^{12}$ Aunque, el componente atribuido a las dotaciones resulta negativo y no significativo.
} 
ocupaciones TIC no reducen significativamente el gap salarial existente por género respecto a su posible situación dentro de las ocupaciones no TIC, sí es cierto que reciben un trato, en general, menos discriminatorio, al disminuir el peso explicativo del componente de coeficientes desde el 94,8 por ciento hasta el 77,0 por ciento.

\section{Conclusiones}

La investigación realizada encuentra su justificación en tres hechos fundamentales. En primer lugar, el importante avance y difusión de las TIC en España, fenómeno reciente pero intenso que está modificando profundamente el mercado de trabajo. En segundo lugar, la persistente observación de desigualdades laborales por razones de género en España. Por último, los potenciales efectos correctores de estas desigualdades que la literatura asocia a la difusión de las TIC. Estos hechos nos han llevado a estudiar en qué medida el empleo más directamente ligado a las TIC es capaz, o no, de modificar uno de los quizás más preocupantes aspectos de la desigualdad laboral de género: la discriminación salarial. Para ello, la estrategia ha consistido en la estimación del componente de las diferencias laborales por género no explicada por las características de los trabajadores (descomposición de Oaxaca), primero para el empleo TIC y no TIC, y posteriormente para mujeres y hombres en cada uno de estos dos conjuntos del empleo. La idea final es comprobar si el empleo TIC, donde se observa que la mujer disfruta de una relativamente mejor situación laboral, tiene capacidad para reducir los habituales niveles de discriminación salarial por género observados.

Los resultados obtenidos apuntan al hecho de que desarrollar ocupaciones asociadas a las TIC supone, en general, percibir unos rendimientos salariales superiores. La diferencia salarial existente en relación al desarrollo de otras tareas se explica aproximadamente en un 48,3 por ciento por la mejor dotación de los trabajadores que desarrollan tareas asociadas a las TIC y en un 51,7 por ciento por la existencia de una mayor remuneración dentro de las TIC per se. Ello puede ser debido a la existencia de un trato diferencial en el mercado.

Por otra parte, las mujeres al desarrollar ocupaciones asociadas a las TIC cobran menos que los hombres en la misma situación. Su gap salarial respecto a los varones en este tipo de empleo es incluso ligeramente mayor en comparación con las mujeres que desarrollan tareas no consideradas TIC (397 euros frente a 350,2 euros, respectivamente) aunque su componente discriminatorio o diferencial 
resulta algo más reducido (305,8 euros frente a 332,0 euros). De esta forma, la discriminación salarial por género se reduce en el empleo TIC en 26,3 euros, en tanto que, para ambos géneros, la diferencia en este componente entre el empleo TIC y no TIC era de 216 euros.

En definitiva, cabe afirmar que el empleo TIC introduce unos pobres resultados en cuanto a la reducción de la discriminación salarial enfrentada por las mujeres en nuestro mercado de trabajo. Frente a las potencialidades atribuidas a las Tecnologías de la Información y la Comunicación en términos de oportunidades laborales para las mujeres y la consecución de mayores niveles de igualad, la explicación podría apuntar al predominio en el mercado de trabajo, también en relación el empleo TIC, de unas profundas, generales y persistentes estructuras diferenciadas por razones de género.

Sobre la base de estas conclusiones, las implicaciones de política económica son claras. La difusión y aplicación de las TIC no se constituye en un instrumento suficiente al que confiar la reducción y eliminación de las desigualdades laborales por género, de manera que la política laboral debe perseverar en el diseño y aplicación de medidas que persigan específicamente este objetivo.

Por último, queremos resaltar el hecho de que, como ha sido mencionado, las estimaciones salariales efectuadas se basan en la definición del sector TIC con una desagregación de la CNAE-93 a 2 dígitos, cuando la acotación original de esta parte del empleo requeriría de un detalle de tres dígitos. Hay que ser consciente de que este hecho puede introducir alguna perturbación en los resultados, aunque éstos, a nuestro juicio, no revisten una excesiva relevancia. A pesar de ello, resulta necesario de cara al desarrollo de análisis más precisos la construcción de estadísticas sobre salarios con un mayor grado de desagregación sectorial, así como quizás nuevas metodologías para definir las TIC con mayor concreción.

\section{Bibliografía}

Acemoglu, D. (2002) "Technical Change, Inequality and the Labor Market”. Journal of Economic Literature, 40, n ${ }^{0}$ 1, 7-72.

Aláez R. y Ullibarri M. (1995) "Discriminación salarial por sexo: Un análisis del sector privado y sus diferencias regionales”. ICE, Tribuna económica. Diciembre 2000-Enero 2001, nº 789, 117-138. 
Ben Jann, 2008. "OAXACA: Stata module to compute the Blinder-Oaxaca decomposition," Statistical Software Components S456936, Boston College Department of Economics, http://repec.ethz.ch/ets/papers/jann_oaxaca.pdf

Blinder, A.S. (1973). "Wage Discrimination: Reduced Form and Structural Estimates”. The Journal of Human Resources, $\mathrm{n}^{\circ}$ 8, 436-455.

Carnoy, M. (2002). Sustaining the New Economy. Work, Family and Community in the Information Age. Cambridge, Massachusetts: Harvard University Press.

Colecchia, A., and Papaconstantinou, G. (1996). "The Evolution of Skills in OECD Countries and the Role of Technology". Science, Technology, Industry (STI) Working Papers, OECD, Paris, nº 1996/8, 1-37.

Chen, Derek H. C. (2004) "Gender Equality and Economic Development. The Role for Information and Communication Technologies" World Bank Policy research Working Paper, $\mathrm{n}^{\circ}$ 3285, April, 1-34.

De la Rica, R. y Ugidos, A. (1995) “¿Son las diferencias en capital humano determinantes de las diferencias salariales observadas entre hombres y mujeres?". Investigaciones Económicas, vol. XIX (3), Septiembre, 395-414.

Diaz-Chao (2008) "Las diferencias salariales en la economía del conocimientos: un análisis empírico para España”. UOC papers. Revista sobre la Sociedad del Conocimiento, $\mathrm{n}^{\circ}$ 6, 1-7. http://uocpapers.uoc.edu.

Ducatel, K. and Burgelman, JC. (1999). "The Futures Project Employment Map”, Joint Research Centre, Brussels: European Commission, Series, $\mathrm{n}^{\circ}$ 3 ,

Dolado, J.J., Felgueroso, F. y Jimeno, J. F. (2002) "Recent Trends in Occupational Segregation by Gender: A Look across the Atlantic". Documentos de trabajo de FEDEA, Abril, vol. 2002-11.

Eurostat (2006). Employment in high technology. Statistics in Focus. Science and Technology. 1/2006.

Figart, D., Mutari, E. and Power, M. (2002). Living Wages, Equal Wages: Gender and Labour Market Policies in the United States. United Kingdom. Routledge.

Heckman, J. J. (1976) "The Common Structure of Statistical Models of Truncation, Sample Selection and Limited Dependent Variables and a Simple Estimator for Such Models". Annals of Economics and Social Measure, n ${ }^{\circ}$ 5, 120-137. 
Heckman, J. J. (1979) "Sample Selection Bias as a Specification Error" Econometrica, n 47, 153-161.

Hernandez, P.J. y Méndez, I. (2005) "La corrección del sesgo de selección en los análisis de corte transversal de discriminación salarial por sexo: estudio comparativo en los países de la Unión Europea”. Estadística española, vol. 47, $\mathrm{n}^{\circ}$ 158, 179-214.

Hernandez, P.J. (1995) "Análisis empírico de la discriminación salarial de la mujer en España”. Investigaciones Económicas, vol. XIX (2), Mayo, 195215.

Iglesias, C. y Llorente, R. (2008) "Evolución reciente de la segregación laboral por género en España". Documento de trabajo del Instituto Universitario Económico y Social (IAES), n ${ }^{\circ}$ 13/2008, http://www.iaes.es.

Iglesias, C., Llorente, R. y Dueñas, D. (2009). "La expansión de los servicios y la transformación del empleo en España". Papeles de Economía Española, 120, 28-43.

Napari, S. (2009) "Gender differences in early-career wage growth" Labour Economic, Elsevier, n $16,140-148$.

Neuman, S., Oaxaca, R.L. (2004). "Wage decompositions with selectivitycorrected wage equations: A methodological note". Journal of Economic Inequality, $\mathrm{n}^{\circ} 2,3-10$.

Neumark, D. (1988). "Employers' Discriminatory Behaviour and the Estimation of Wage Discrimination". The Journal of Human Resources, $\mathrm{n}^{\mathrm{o}}$ $23,279-295$.

Oaxaca, R. (1973). "Male-Female Wage Differentials in Urban Labour Markets". International Economic Review. n ${ }^{\circ}$ 14, 693-709.

Oaxaca, R.L., Ransom, M.R. (1999). "Identification in Detailed Wage Decompositions". The Review of Economics and Statistics, n ${ }^{\circ}$ 81, 154-7.

Oaxaca, R.L., Ransom, M.R. (1994). "On discrimination and the decomposition of wage differentials". Journal of Econometrics, n ${ }^{0}$ 61, 5-21.

OCDE (2002). The Measurement of Scientific and Technological Activities. Frascati Manual 2002. OCDE. París.

OCDE (2004). OECD Information Technology Outlook, 2004. OCDE. Paris.

OECD (2007) "ICTs and Gender". OECD Digital Economy Papers, OECD publishing, $\mathrm{n}^{\circ} 129,1-45$. 
OIT (2001) Informe sobre el empleo en el mundo. La vida en el trabajo de la economía de la información. http://www.ilo.org.

Plasman, R. y Sissomo, S. (2004) "Comparing apples with oranges. Revisiting the gender wage gap in an international perspective" Discussion paper series - IZA, $\mathrm{n}^{\circ} 1.449$, December.

Simon, H. (2006) "Diferencias salariales entre hombres y mujeres en España: Una comparación internacional con datos emparejados empresatrabajador". Investigaciones Económicas, Vol. XXX, 55-87.

Torrent-Sellens, J. (2008) "Cambio tecnológico sesgador de habilidades (eSBTC), ocupación y salarios: un estado de la cuestión”. UOC papers. Revista sobre la Sociedad del Conocimiento, $\mathrm{n}^{\circ} \quad 6,1-14$. http://uocpapers.uoc.edu.

WWW-ICT (2004). Conceptual framework and state of the art. Widening Women's Work in Information and Communication Technology. wwwICT.com. 


\section{Anexo}

Cuadro A.1. Composición del empleo TIC según diferentes definiciones y fuentes en base a las ocupaciones relacionadas con las TIC. Definición a 3 y 2 dígitos. (Fuente: EPA, segundo trimestre de 2007 y ECV-2008).

\begin{tabular}{|c|c|c|c|}
\hline \multicolumn{2}{|c|}{ EPA, segundo trimestre de 2007} & \multicolumn{2}{|c|}{ ECV-2008 } \\
\hline CNO-94 & $\begin{array}{l}\text { Ocupaciones Laborales } \\
\text { (Definición a } 3 \text { dígitos) }\end{array}$ & CNO-94 & $\begin{array}{l}\text { Ocupaciones Laborales } \\
\text { (Definición a } 2 \text { dígitos) }\end{array}$ \\
\hline 111 & Dirección general y presidencia ejecutiva & 11 & $\begin{array}{l}\text { Dirección de empresas de } 10 \text { ó más } \\
\text { asalariados }\end{array}$ \\
\hline 112 & Dirección de departamento de producción & & \\
\hline 113 & $\begin{array}{l}\text { Dirección de áreas y departamento } \\
\text { especializados }\end{array}$ & & \\
\hline $\begin{array}{l}201 \\
261\end{array}$ & Físicos, químicos y asimilados & 20 & $\begin{array}{l}\text { Profesiones asociadas a titulaciones de } 2^{\circ} \text { y } \\
\text { 3er ciclo universitario en oc. físicas, } \\
\text { químicas, matemáticas y asimilados }\end{array}$ \\
\hline $\begin{array}{l}202 \\
262\end{array}$ & $\begin{array}{l}\text { Mat emáticos, act uarios, estadísticos y } \\
\text { asimila dos }\end{array}$ & 23 & Profesionales del derecho \\
\hline $\begin{array}{l}203 \\
263\end{array}$ & $\begin{array}{l}\text { Profesionales de la informática de nivel } \\
\text { superior }\end{array}$ & 24 & $\begin{array}{l}\text { Profesionales en organizaciones de empresas, } \\
\text { profesionales en las cc. Sociales y hum anas } \\
\text { asociadas a titulaciones de } 2^{\circ} \text { y } 3 \text { er ciclo uni. }\end{array}$ \\
\hline $\begin{array}{l}204 \\
264\end{array}$ & $\begin{array}{l}\text { Arquitectos, urbanistas, e ing enieros } \\
\text { planificación del tráfico }\end{array}$ & 25 & $\begin{array}{l}\text { Escritores, artistas y otras profesiones } \\
\text { asociadas }\end{array}$ \\
\hline $\begin{array}{l}205 \\
265\end{array}$ & Ingenieros superiores y técnicos & 26 & $\begin{array}{l}\text { Profesione s asociadas a una titulación de ler } \\
\text { ciclo uni. en cc. físicas, químicas, matemáticas, } \\
\text { ingeniería y asimilados }\end{array}$ \\
\hline 231 & Abogados y fiscales; jueces y magistrados & 29 & $\begin{array}{l}\text { Otras profesiones asocia das a una titulación } \\
\text { de 1er ciclo universitaria }\end{array}$ \\
\hline 239 & Otros profesionales del Derecho & & \\
\hline $\begin{array}{l}241 \\
291 \\
252 \\
292\end{array}$ & $\begin{array}{l}\text { Profesionales en administración y dirección } \\
\text { de empresa } \\
\text { Archiveros, bibliotecarios y profesionales } \\
\text { asimilados }\end{array}$ & & \\
\hline 303 & Profesionales técnicos de la informática & 30 & $\begin{array}{l}\text { Técnicos de las ciencias físicas, químicas e } \\
\text { ingenierías }\end{array}$ \\
\hline 304 & Operadores de equipos ó pticos y electrónicos & 34 & $\begin{array}{l}\text { Profesionales de a poyo a la gestión } \\
\text { administrativa }\end{array}$ \\
\hline 331 & $\begin{array}{l}\text { Profesionales a poyo en operaciones } \\
\text { financieras y comerciales }\end{array}$ & 35 & Otros técnicos y profesionales de apoyo \\
\hline 332 & $\begin{array}{l}\text { Representantes de comercio y técnicos de } \\
\text { venta }\end{array}$ & & \\
\hline 341 & $\begin{array}{l}\text { Profesionales a poyo gestión adtva. Con tareas } \\
\text { adtvas. Grales. }\end{array}$ & & \\
\hline 351 & $\begin{array}{l}\text { Consignatarios y ag entes en la contratación } \\
\text { de mano de obra }\end{array}$ & & \\
\hline 401 & Auxiliares contables y financieros & 40 & $\begin{array}{l}\text { Empleados de oficina de ss. estadísticos, } \\
\text { financieros y bancarios }\end{array}$ \\
\hline 421 & $\begin{array}{l}\text { Taquígrafos y mecanógrafos, graba dores de } \\
\text { datos }\end{array}$ & 42 & Operadores de máquinas de oficina \\
\hline 430 & $\begin{array}{l}\text { Auxiliares adtvos. Sin tareas de atención al } \\
\text { publico n.c.a. }\end{array}$ & 43 & $\begin{array}{l}\text { Aux. administrativos sin tareas de atención al } \\
\text { público no clasificados a nteriormente }\end{array}$ \\
\hline 440 & $\begin{array}{l}\text { Auxiliares adtvos. Con tareas de atención al } \\
\text { publico n.c.a. }\end{array}$ & 44 & $\begin{array}{l}\text { Aux. administrativos con tareas de at ención } \\
\text { al público no clasifica dos anteriormente }\end{array}$ \\
\hline 762 & $\begin{array}{l}\text { Mecánicos y ajustadores equipos eléctricos y } \\
\text { electrónicos }\end{array}$ & 76 & $\begin{array}{l}\text { Mecánicos y ajusta dores de maquinaria y } \\
\text { equipos eléctricos y electrónicos }\end{array}$ \\
\hline
\end{tabular}


Cuadro A.2. Características del empleo en ocupaciones TIC vs. Resto de ocupaciones. Porcentajes e incidencia. (Fuente: EPA, $2^{\circ}$ trimestre de 2007).

\begin{tabular}{|c|c|c|c|c|c|}
\hline \multicolumn{2}{|c|}{ Características } & Ocup. TIC & Resto & $\begin{array}{c}\text { Incidencia } \\
\text { (Ocup. TIC } \\
\text { sobre } \\
\text { Resto) }\end{array}$ & $\begin{array}{c}\text { Incidencia } \\
\text { (Ocup. TIC } \\
\text { sobre el } \\
\text { empleo total) }\end{array}$ \\
\hline \multirow{3}{*}{ Edad } & Jóvenes & 42,7 & 42,6 & 1,0 & 1,0 \\
\hline & Intermedios & 47,2 & 46,0 & 1,0 & 1,0 \\
\hline & Maduros & 10,2 & 11,4 & 0,9 & 0,9 \\
\hline \multirow{2}{*}{ Estado civil } & Soltero/a & 35,7 & 36,3 & 1,0 & 1,0 \\
\hline & No soltero/a & 64,3 & 63,7 & 1,0 & 1,0 \\
\hline \multirow{3}{*}{ Estudios } & Primarios & 2,6 & 18,2 & 0,1 & 0,2 \\
\hline & Secundarios & 38,2 & 56,1 & 0,7 & 0,7 \\
\hline & Superiores & 59,2 & 25,7 & 2,3 & 1,8 \\
\hline \multirow{2}{*}{$\begin{array}{l}\text { Sector de } \\
\text { actividad }\end{array}$} & Actividades TIC & 9,8 & 1,0 & 10,1 & 3,4 \\
\hline & Resto actividades & 90,2 & 99,0 & 0,9 & 0,9 \\
\hline \multirow{2}{*}{$\begin{array}{l}\text { Situación } \\
\text { profesional }\end{array}$} & Asalariados/as & 84,2 & 81,9 & 1,0 & 1,0 \\
\hline & No asalariados/as & 15,8 & 18,1 & 0,9 & 0,9 \\
\hline \multirow{2}{*}{$\begin{array}{l}\text { Tipo de } \\
\text { contrato }\end{array}$} & Indefinido & 82,9 & 64,1 & 1,3 & 1,2 \\
\hline & Temporal & 17,1 & 35,9 & 0,5 & 0,5 \\
\hline \multirow{2}{*}{$\begin{array}{l}\text { Tipo de } \\
\text { jornada }\end{array}$} & Completa & 91,7 & 87,0 & 1,1 & 1,2 \\
\hline & Parcial & 8,3 & 13,0 & 0,6 & 0,5 \\
\hline
\end{tabular}

Cuadro A.3. Estadísticos de las variables utilizadas en las ecuaciones salariales (cuadro 4 y 6). (Fuente: elaboración propia sobre datos de la ECV-2008, INE).

\begin{tabular}{|c|c|c|c|c|c|c|c|c|}
\hline \multirow[b]{2}{*}{ Variable } & \multicolumn{4}{|c|}{$\begin{array}{c}\text { Estimación salarial recogida en el } \\
\text { cuad ro } 4\end{array}$} & \multicolumn{4}{|c|}{$\begin{array}{c}\text { Estimación salarial recogida en el } \\
\text { cuadro } 6\end{array}$} \\
\hline & Media & $\begin{array}{l}\text { Desv. } \\
\text { Típica }\end{array}$ & Min. & Max. & Media & $\begin{array}{l}\text { Desv. } \\
\text { Típica }\end{array}$ & Min. & Max. \\
\hline $\mathrm{W}$ & $1.830,66$ & 977,99 & 200,00 & 14345,33 & 2170,71 & 1048,23 & 300,00 & 14345,33 \\
\hline $\ln W$ & 7,40 & 0,45 & 5,30 & 9,57 & 7,59 & 0,44 & 5,70 & 9,57 \\
\hline Casado & 0,56 & 0,50 & 0,00 & 1,00 & 0,57 & 0,50 & 0,00 & 1,00 \\
\hline Español & 0,93 & 0,25 & 0,00 & 1,00 & 0,98 & 0,16 & 0,00 & 1,00 \\
\hline Estud. Sec. 1ra. Etapa & 0,23 & 0,42 & 0,00 & 1,00 & 0,07 & 0,25 & 0,00 & 1,00 \\
\hline Estud. Sec. 2da. Etapa & 0,24 & 0,43 & 0,00 & 1,00 & 0,21 & 0,41 & 0,00 & 1,00 \\
\hline FP e inserción laboral & 0,01 & 0,09 & 0,00 & 1,00 & 0,01 & 0,07 & 0,00 & 1,00 \\
\hline Estudios superiores & 0,40 & 0,49 & 0,00 & 1,00 & 0,70 & 0,46 & 0,00 & 1,00 \\
\hline Experiencia potencial & 19,77 & 11,90 & 0,00 & 63,00 & 18,00 & 11,18 & 0,00 & 54,00 \\
\hline Experiencia potencial $^{2}$ & 532,55 & 557,19 & 0,00 & 3969,00 & 448,87 & 486,28 & 0,00 & 2916,00 \\
\hline Horas de trabajo & 40,85 & 6,59 & 6,00 & 99,00 & 39,02 & 6,24 & 8,00 & 84,00 \\
\hline Empr. de 1 a 10 trab. & 0,28 & 0,45 & 0,00 & 1,00 & 0,24 & 0,42 & 0,00 & 1,00 \\
\hline Empr. de 20 a 49 trab. & 0,15 & 0,36 & 0,00 & 1,00 & 0,18 & 0,39 & 0,00 & 1,00 \\
\hline Empr. de 50 o más trab. & 0,37 & 0,48 & 0,00 & 1,00 & 0,41 & 0,49 & 0,00 & 1,00 \\
\hline Tem poral & 0,24 & 0,43 & 0,00 & 1,00 & 0,19 & 0,39 & 0,00 & 1,00 \\
\hline Obs. no ponderadas & \multicolumn{4}{|c|}{$9.950,0$} & \multicolumn{4}{|c|}{$2.171,00$} \\
\hline $\begin{array}{l}\text { Obs. ponderadas } \\
\text { (miles de personas) }\end{array}$ & \multicolumn{4}{|c|}{$13.866,0$} & \multicolumn{4}{|c|}{$3.181,70$} \\
\hline
\end{tabular}


Cuadro A.4. Ecuaciones de salarios para los hombres y mujeres que desarrollan una ocupación no considerada como TIC en su puesto de trabajo. (Fuente: elaboración propia sobre datos de la ECV-2008, INE).

\begin{tabular}{|c|c|c|c|c|c|c|}
\hline \multirow[b]{2}{*}{$\ln W$} & \multicolumn{3}{|c|}{ Hombres } & \multicolumn{3}{|c|}{ Mujeres } \\
\hline & Coef. & Error Est. & $\mathbf{P}>\mathbf{Z}$ & Coef. & Error Est. & $\mathbf{P}>\mathbf{z}$ \\
\hline Cas ado & 0,167 & 0,016 & $0,000 * *$ & 0,068 & 0,019 & $0,000^{* *}$ \\
\hline Español & 0,106 & 0,023 & $0,000 * *$ & 0,147 & 0,030 & $0,000 * *$ \\
\hline Estudios de secundaria 1ra. Etapa & 0,066 & 0,017 & $0,000^{* *}$ & 0,078 & 0,027 & $0,004^{* *}$ \\
\hline Es tudios de secundaria 2da. Etapa & 0,192 & 0,019 & $0,000^{* * *}$ & 0,244 & 0,028 & $0,000^{* *}$ \\
\hline FP e inserción laboral & 0,293 & 0,051 & $0,000 * *$ & 0,226 & 0,053 & $0,000^{* *}$ \\
\hline Es tudios superiores & 0,432 & 0,021 & $0,000^{* *}$ & 0,536 & 0,031 & $0,000^{* *}$ \\
\hline Experiencia poten cial & 0,013 & 0,002 & $0,000^{* *}$ & 0,013 & 0,003 & $0,000^{* *}$ \\
\hline Experiencia poten $\mathrm{cial}^{2}$ & 0,000 & 0,000 & $0,000 * *$ & 0,000 & 0,000 & $0,001^{* *}$ \\
\hline Horas de trabajo & 0,008 & 0,001 & $0,000 * *$ & 0,009 & 0,002 & $0,000^{* *}$ \\
\hline Empresa de 11 a 19 trabajadores & $-0,025$ & 0,017 & 0,130 & $-0,118$ & 0,025 & $0,000^{* *}$ \\
\hline Empresa de 20 a 49 trabajadores & 0,079 & 0,021 & $0,000 * *$ & 0,008 & 0,031 & 0,788 \\
\hline Empresa de 50 o más trabajadores & 0,178 & 0,016 & $0,000 * *$ & 0,148 & 0,025 & $0,000 * *$ \\
\hline Temporal & $-0,136$ & 0,016 & $0,000 * *$ & $-0,139$ & 0,019 & $0,000 * *$ \\
\hline Constante & 6,493 & 0,064 & $0,000 * *$ & 6,261 & 0,090 & $0,000 * *$ \\
\hline $\mathrm{N}^{0}$ de obs ervaciones no censuradas & & 4.968 & & & 2.811 & \\
\hline $\mathrm{R}^{2}$ & & 0,370 & & & 0,367 & \\
\hline Sig. & & 0,000 & & & 0,000 & \\
\hline
\end{tabular}

** Significativo bajo una probabilidad del $95 \%$.

*Significativo bajo una probabilidad del $90 \%$.

Cuadro A.5. Descomposición de Oaxaca sobre el diferencial de salarios existente entre hombres y mujeres que trabajan en ocupaciones no consideradas como TIC. (Fuente: elaboración propia sobre datos de la ECV-2008, INE).

\begin{tabular}{|c|c|c|c|c|c|}
\hline \multirow[b]{2}{*}{ Diferencial } & \multicolumn{4}{|c|}{ Descomposición del LnW } & \multirow[t]{2}{*}{ Valores monetarios } \\
\hline & Coef. & Std. Err. & $\mathrm{P}>\mathrm{z}$ & $\%$ & \\
\hline Predicción del lnW en TIC-Hombres & 7,431 & 0,008 & $0,000 * *$ & & $1.686,9$ \\
\hline Predicción del $\operatorname{lnW}$ en TIC-Mujeres & 7,198 & 0,011 & 0,000 ** & & $1.336,7$ \\
\hline Diferencia de $\ln W$ & 0,233 & 0,013 & $0,000 * *$ & & 350,2 \\
\hline \multicolumn{6}{|l|}{ Descomposición } \\
\hline Dotaciones & 0,002 & 0,010 & 0,839 & 0,9 & 3,1 \\
\hline Coeficientes & 0,221 & 0,012 & $0,000 * *$ & 94,8 & 332,0 \\
\hline Interacción (variables y coeficientes) & 0,010 & 0,007 & 0,167 & 4,3 & 15,1 \\
\hline
\end{tabular}

** Significativo bajo una probabilidad del $95 \%$.

*Significativo bajo una probabilidad del $90 \%$. 